\title{
EDITORIAL
}

\section{TAMING THE DOUBLE-EDGED SWORD}

\author{
Proteolysis, once thought to be simply a degradative pathway for protein destruction, turns out \\ to be central to the regulation of myriad cellular processes. The latest Horizon Symposium got \\ to grips with the field's many outstanding questions, and asked whether we know enough to \\ manipulate these systems to our advantage.
}

The four out of five would-be drugs that fail to make it through clinical trials are a constant reminder of how little we understand the complexity of disease biology. Add to this the fact that there were apparently 3,900 active clinical trials ongoing last year (see the news story on the cost of clinical trials on page 851), and the everexpanding heap of failures takes on an even more vivid character. One area in which the surprises that lie in wait for us when we try to tamper with the workings of biological systems have been rather shockingly revealed is the world of proteolysis. Inhibiting proteolysis has certainly led to some very notable victories — inhibitors of HIV protease and angiotensin-converting enzyme (ACE) being striking examples of success — but has more often given rise to development programmes that encountered unexpected barriers. Matrix metalloproteinases and caspases, two classes of proteases selected for inhibition in cancer and conditions involving apoptosis, respectively, have been the intended target of a particularly depressing number of failed compounds.

So what better subject than proteolysis to concentrate on in the third Horizon Symposium, 'Signalling scissors: new perspectives on proteases', which took place at the end of last month. These small, three-day discussion meetings, which we launched last year in collaboration with Aventis, bring a broad group of scientists together in a retreat location to discuss the key questions in an exciting and fast-moving area of biomedical science. They aim to get people talking across boundaries, both across disciplines and across the academic-industrial divide. As always, background articles, summaries of the questions discussed, a list of participants and highlights of the meeting are available for free on the dedicated website at www.horizonsymposia.com.

Many of the attendees at the meeting asked what we have learnt from the numerous clinical failures, and certainly standing atop the 'heap' should afford us a clearer overview of the subject. Proteases are the largest class of enzymes and, with more than 500 genes encoding proteases predicted to exist in the mammalian genome, are also among the most abundant of all gene products. Not surprisingly, given nature's huge investment in different forms of protease, the traditional view of proteolytic enzymes as being purely part of a nonspecific degradative machinery is being overturned. We are now coming to realize the much more refined ways in which proteolysis regulates living processes. Rather than the all-or-nothing destruction of functional proteins and peptides, proteolytic processing of biomolecules can be used to fine-tune cellular and extracellular processes, by producing regulated amounts of activated or deactivated products. Such modulatory control is being observed in several areas of biology, including wound healing, cell proliferation, angiogenesis and apoptosis, and the list of fields in which proteases have a key role is still growing rapidly.

Alongside our advances in amassing an understanding of the full set of proteases and their protein substrates, we are also beginning to learn about the regulation of proteolytic function by endogenous enzyme inhibitors and activators. In turn, insights into their modes of operation are opening up the possibility of developing new tools to regulate proteases selectively. Given the demonstrated role of disrupted proteolytic processing in many disease states, the need for new methods of controlling protease function is particularly pressing. But regulating proteolytic activity has required nature to develop a particularly sophisticated intra- and extracellular toolbox, and will probably require us to do the same if we want to exploit these systems. Harnessing the power of proteases to good effect will therefore test our ingenuity to the full, and understanding the complexity of proteolytic systems represents exactly the sort of challenge that the Horizon Symposia were created to address. 